\title{
Erratum: Integrated multi-omics of the human gut microbiome in a case study of familial type 1 diabetes
}

Anna Heintz-Buschart, Patrick May, Cédric C. Laczny, Laura A. Lebrun, Camille Bellora, Abhimanyu Krishna, Linda Wampach, Jochen G. Schneider, Angela Hogan, Carine de Beaufort and Paul Wilmes

Nature Microbiology 2, 16180 (2016); published 10 October 2016; corrected 24 October 2016

This Article should have been published under a Creative Commons licence according to the Nature policy on publishing the primary sequence of an organism's genome for the first time. The editors apologize to the authors and to readers for this error. The manuscript is now open access and published under a CC-BY licence. All versions of the Article have been modified accordingly. 